\title{
Mäßiger Marihuanakonsum ist nicht schädlich
}

\section{Die Substanzen des Marihuana- und des Tabakrauchs ähneln sich. Bisher waren Daten zum Einfluss des Marihuanakonsums auf die Lungenfunktion dennoch widersprüchlich. Eine große Kohortenstudie von M. J. Pletcher et al. zeigt jetzt deutliche Unterschiede der Effekte von Tabak- und Marihuanakonsum auf forciertes Einsekundenvolumen ( $\left.\mathrm{FEV}_{1}\right)$ und Vitalkapazität (FVC). JAMA 2013; 307: 173-181}

Basis der Auswertung war die longitudinale CARDIA-Untersuchung (Coronary Artery Risk Development in Young Adults Study). In dieser wurden im Zeitraum von 1985-2006 bei 5115 Männern und Frauen in 4 Städten in den USA wiederholt Lungenfunktionsmessungen durchgeführt und Daten zu Rauch- und Lebensgewohnheiten erhoben. Ein Drittel der Probanden rauchte gelegentlich oder reposition mit Marihuana wurde in JointJahren bemessen, wobei ein Joint-Jahr 365 gerauchten Joints oder MarihuanaPfeifen entsprach. Die Teilnehmer der Studie waren zu Beginn der Erhebung zwischen 18 und 30 Jahren alt. Ähnlich viele Teilnehmer konsumierten Tabak und Marihuana. Allerdings war der Marihuanakonsum mit im Median 2-3 Gele-

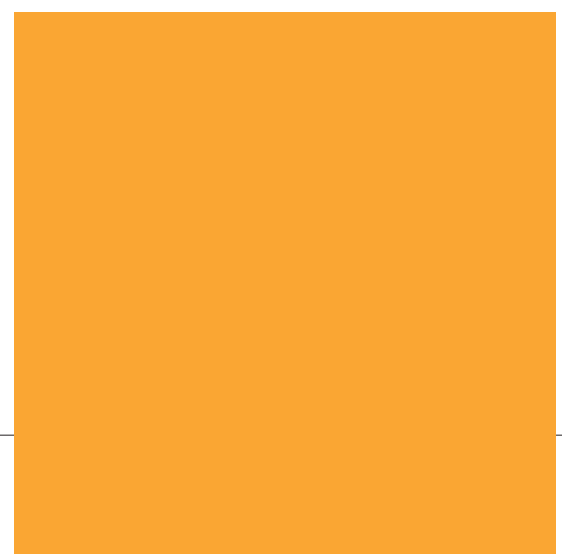

\section{Überraschend positive Effekte} $\nabla$

Wie zu erwarten, war die Tabakexposition, sowohl aktuell als auch über die Lebenszeit hinweg, linear assoziiert mit einer erniedrigten FEV $_{1}$ und FVC. Der Tabgelmäßig Marihuana. Die Lebenszeitexgenheiten pro Monat meist gering.

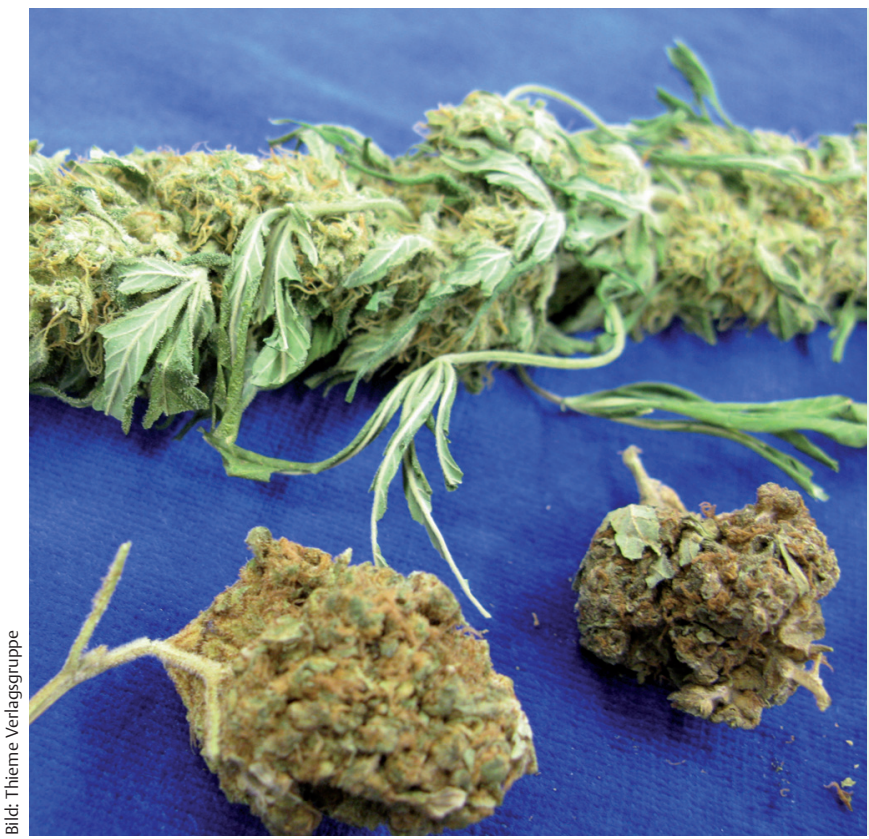

akkonsum stellte damit eine positive Kontrolle für die Messungen dar. Die Assoziation zwischen Marihuanaexposition und Lungenfunktion war aber nicht wie beim Tabakrauchen linear: Bei niedrigerer Exposition ließ sich keine Verschlechterung, sondern eine Verbesserung der Lungenfunktion nachweisen: Der $\mathrm{FEV}_{1^{-}}$ Wert nahm bei leichtem Marihuanakonsum um $13 \mathrm{ml}$ pro Joint-Jahr zu (95\% Konfidenzintervall KI 6,4 bis 20; p<0,001), die FVC um $20 \mathrm{ml}$ pro Joint-Jahr (95\% KI, 12 bis 27; $\mathrm{p}<0,001$ ).

Bei höherem Expositionsniveau reduzierte sich die Assoziation und schien sich sogar, ins Negative zu verkehren: Bei mehr als 10 Joint-Jahren nahm die $\mathrm{FEV}_{1}$ um $-2,2 \mathrm{ml}$ pro Joint-Jahr ab (95\% KI -4,6 bis 0,$3 ; \mathrm{p}=0,08$ ) und um $-3,2 \mathrm{ml}$ pro Marihuanakonsum/Monat ( $95 \% \mathrm{KI}-5,8$ bis $-0,6$; $\mathrm{p}=0,02$ ) bei mehr als 20 Konsumepisoden pro Monat. Mit sehr hohem Marihuanakonsum unterschied sich die Assoziation von $\mathrm{FEV}_{1}$ aber nicht vom Ausgangswert. Die FVC blieb deutlich höher als bei der Ausgangsmessung bei Beobachtungsbeginn (bei 20 Joint-Jahren 76 ml; 95\% KI 34 bis 117; $\mathrm{p}<0,001)$. Allerdings war die Zahl der Personen mit umfangreichem Marihuanakonsum zu gering, um für diese Gruppe sichere Aussagen zu treffen.

\section{Tiefes Inhalieren gut für die Lungenfunktion?}

$\nabla$

Die Autoren spekulieren, ob das tiefe Inhalieren beim Haschischrauchen ein Grund für die Verbesserung der Lungenfunktion oder auch nur eine bessere Performance bei der Lungenfunktionsmessung sein könnte. Die Untersuchungen sprechen jedenfalls dafür, dass ein gelegentlicher Konsum von Marihuana, z.B. aus medizinischen Gründen bei Schmerzen oder Appetitverlust, nicht mit einer Verschlechterung der Lungenfunktion einhergehen muss.

\section{Fazit}

Gelegentlicher oder geringer Marihuanakonsum war in dieser großen Longitudinalstudie nach Angaben der Autoren nicht mit einer Verschlechterung der Lungenfunktion assoziiert, sondern sogar mit einer Verbesserung derselben.

Friederike Klein, München 